\title{
Design, Cultivation and Application of Load-Bearing Mycelium Components: The MycoTree at the 2017 Seoul Biennale of Architecture and Urbanism
}

\author{
Felix Heisel $^{1}$, Juney Lee ${ }^{2}$, Karsten Schlesier ${ }^{1}$, Matthias Rippmann ${ }^{2}$, Nazanin Saeidi ${ }^{3}$, \\ Alireza Javadian ${ }^{3}$, Adi Reza Nugroho ${ }^{4}$, Tom Van Mele ${ }^{2}$, Philippe Block ${ }^{2}$, Dirk E. Hebel ${ }^{1}$ \\ ${ }^{1}$ Sustainable Construction, Faculty of Architecture, KIT Karlsruhe, Karlsruhe, Germany, \\ ${ }^{2}$ Block Research Group, Department of Architecture, ETH Zürich, Zurich, Switzerland, \\ ${ }^{3}$ Alternative Construction Materials, Future Cities Laboratory, Singapore-ETH Centre, \\ Singapore \\ ${ }^{4}$ Mycotech, PT Miko Bahtera Nusantara, Indonesia
}

\begin{abstract}
MycoTree is a spatial branching structure made out of load-bearing mycelium components. Its geometry was designed using $3 D$ graphic statics, utilising compression-only form to enable the weak material to perform structurally. Using only mycelium and bamboo, the structure represents a provocative vision of how one may move beyond the mining of our construction materials from the earth's crust to their cultivation; how achieving stability through geometry rather than through material strength opens up possibilities to use weaker materials structurally and safely; and ultimately, how newly developed cultivated materials in combination with informed structural design have the potential to propose an alternative to established building materials for a more sustainable construction industry.
\end{abstract}

\section{Introduction}

As populations and urban aspirations grow, so does the demand for resources to support them. Although such demands were once satisfied by local and regional hinterlands, they are becoming increasingly global in scale and reach. This phenomenon has generated material flows that are trans-continental in scope and has profound consequences for the sustainability, functioning, sense of ownership and identity of future cities. However, the focus of the global construction industry on only a few established building materials results in a concentrated consumption of specific and finite natural resources [1]. In this respect, the built environment of the future city demands the utilisation of alternative resources.

The 21 st century will face a radical paradigm shift in how materials are produced for the construction of future habitats. The linear practice of 'take, make and dispose' has proven itself unsustainable in the face of depleting resources and exponentially growing urban populations [2]. Instead, alternative materials and approaches to construction must be investigated to move towards a cycle of 'take, make, and repeat'. Materials that were previously considered unwanted and low-strength may present possibilities to address this undesirable state of affairs.

MycoTree, the prototypical structure described in this paper, consists of organic building components made from agricultural waste, which are stabilized only by the grown matrix of mushroom mycelium. Its geometry was designed using 3D graphic statics, utilising compression-only form to enable the weak material to perform structurally. The resulting expressive and provocative structure demonstrates that the combination of state-of-the-art research in cultivated building materials - which can be effectively grown locally or even on-site - with informed structural design and engineering may bring about the changes that are desperately needed.

\section{Depleting finite resources}

Over the last few centuries, the global construction industry has grown increasingly more dependent on mined resources. Today, steelreinforced concrete is the most produced construction material on an industrial scale worldwide, with more than $50 \%$ of all man-made objects containing cement-bearing materials [3].

Mining, in contrast to cultivating, has a significant disadvantage: excavated materials do not regenerate themselves. The aggregates used in a concrete mix, e.g. sand and gravel, are forever lost and transformed without the possibility to recover them in their original form. In the case of sand, the ruthless exploitation of this natural resource already shows dramatic consequences.

Sand is the product of millions of years of natural decomposition of rocks from mountainous areas that is flushed through streams and rivers into the oceans. Yet, the ever-growing building industry mines this 
natural resource at an alarmingly unsustainable rate; today, twice as much sand is being consumed as it is produced naturally in the same amount of time [4]. The global market for sand is estimated at 15 to 30 billion tonnes per year, with a value of more than 70 billion US Dollars [5, 6]. Drastic forms of sand mining are appearing all around the globe: the sands of North African beaches are being illegally harvested, with one out of two beaches in Morocco affected; rivers are being dredged and ocean floors scraped; and landmasses are collapsing and islands are eroding away. The consequences reach far beyond the actual mining areas and leave behind devastating traces. Water levels fall in dredged rivers of India, Thailand, and Cambodia, destroying traditional settlements and their modes of life. Dredging seafloors not only harms the local ecosystems, but also has lasting effects on distant sea regions with sediments suspended in the ocean currents. Marine sand mining has irreversible ramifications that will haunt generations to come. Sand shortages lead to increasing illegal mining practices and trade in developing countries [7].

Other finite minerals and metals in the earth's crust, are equally affected by today's mining-centric standards and practices of the building industry. In 2005, the German Federal Ministry of Economic Affairs and Energy (BMWi) prognosticated that the global reserves of lead, zinc and tin will be depleted in less than 25 years with then-current rate of mining and consumption [1]. Even copper and iron deposits are in danger of depletion if today's 'take, make and dispose' mentality continues [8]. At the same time, global material extraction has more than doubled in the past 30 years, and is estimated to continue rising: from 65 billion metric tons of raw materials entering the economic system in 2010 to about 82 billion metric tons in 2020 [9].

In recent decades, the building industry has reacted to this increasing scarcity of mined resources by aiming for a reduction of construction material use through higher efficiency. An alternative pathway is being presented by the concept of circular economy, a framework 'that is restorative and regenerative by design and aims to keep products, components, and materials at their highest utility and value at all times, distinguishing between technical and biological cycles [10]'. By closing the loop, the circular economy aims to recirculate material resources that are currently being diverted to and lost in landfills, oceans or through incineration.

\section{From linear to circular material application}

In regard to the building industry, the circular economy's design imperative relates to a key requirement: building components must be designed, manufactured, and constructed so that all components used can be disassembled, separated and cleanly returned into their respective technical or biological material cycles to maximise the recovery and reuse of construction materials after a building's life cycle. This may necessitate the redesign of standard building components, the development of innovative new joining systems or an avoidance of glues and un-recyclable composites. The past 40 years have seen the development of various design guidelines and manuals in an effort to move towards a more sustainable building environment and practice. Notable examples include Walter R. Stahel's Performance Economy [11], Werner Sobek's Triple Zero [12] guidelines or William McDonough and Michael Braungart's Cradle to Cradle [13]. In these guidelines, materials are considered as borrowed goods, which the client only uses for a certain amount of time and then gives back to the construction market or the natural environment to be reused, recycled, or decomposed.

The development of today's high-performance materials and their assembly are still largely based on the linear economic model, typically aiming to decrease material use by increasing the component's strength. A common result are irreversible (and often non-recyclable) material composites. The paradigm shift towards a circular economy thus not only requires a rethinking of material selection and assembly, but also a new approach towards their structural application, which fully activates the potential of circular recycled or cultivated materials despite their sometimes more challenging material properties. MycoTree addresses both of these aspects by activating a weak material through informed structural design. The utilised new generation of cultivated building materials based on mycelium as a self-assembling glue certainly adapts the above described circular economic model; a building that is built from organically grown materials, also can be composted after its initial use and become the source for a new cultivation and building cycle.

\section{Application of weak materials through informed structural design}

The Professorship of Sustainable Construction at the Karlsruhe Institute of Technology (KIT), the Block Research Group (BRG) at the Swiss Federal Institute of Technology (ETH) Zürich, and the Future Cities Laboratory (FCL) in Singapore are combining their expertise in construction, structural geometry and materials to discover such new design opportunities of natural, reclaimed and cultivated materials to challenge the status quo. While the teams at KIT and FCL investigate alternative, circular building materials and their applicability in the built environment [2, 14], the BRG develops novel structural design techniques to explore compression-only, spatial structures. The advent of 
advanced computational tools, in conjunction with state-of-the-art research in recycled or cultivated materials, has the potential to significantly improve the relevance and applicability of humble, often lowstrength materials in designing of efficient and expressive structures.

As previous collaborations of the authors have demonstrated, materials' weaknesses can be turned into strengths by strategically placing them in an appropriately designed structural geometry. Through informed structural design methods, BRG's research has shown how compression-only structures can empower weak materials to become load-bearing elements at an architectural scale. Compression-only structures significantly reduce the amount of internal stresses, and subsequently reduce the amount of material required to carry the applied loads.

In developing contexts where resources, infrastructure and funds are often inadequate in supply, designing with locally available materials can be a solution for the global scarcity of conventional building materials, by capitalizing upon what is usually abundantly available: manpower. When building with locally-sourced materials like soil-pressed tiles, workers from the community can be trained to make and install the tiles, as in the SUDU - Sustainable Urban Dwelling Unit in Addis Ababa, Ethiopia a collaboration between the Ethiopian Institute of Architecture, Building Construction and City Development EiABC, Dirk E. Hebel and Philippe Block [15]. Shell structures for the ceilings and the roofs with innovative design elements such as stabilizing fins, drastically reduce the need to import and transport expensive, engineered materials because the compression-only structure has been designed for weak materials with low tensile capacity.

In a more urban context, building with recycled materials reduces the rate at which finite resources are being depleted. For the 2015 IDEAS CITY Festival in New York City, the teams of ETH Zürich's Professorship Hebel and the BRG designed and constructed a temporary vault structure built from compressed tetra-pack panels. The shape of the vault, which spanned a neighbourhood park between two buildings in lower Manhattan, followed the flow of forces and was optimised so that the stresses in the structure were predominantly compressive, enabling a considerably weak product - essentially made from discarded beverage containers - to act as a structural material. Following the festival, the panels and other materials used were recycled or reused [16].

Research in development and application of cultivated materials is the next step in these collaborative investigations. As we learn more about their properties and undiscovered potentials, materials that were previously deemed undesirable because they were perceived as vernacular (e.g. bamboo) or even as hazardous waste (e.g. mycelium) present new possibilities for applications in efficient, compression-only structures.

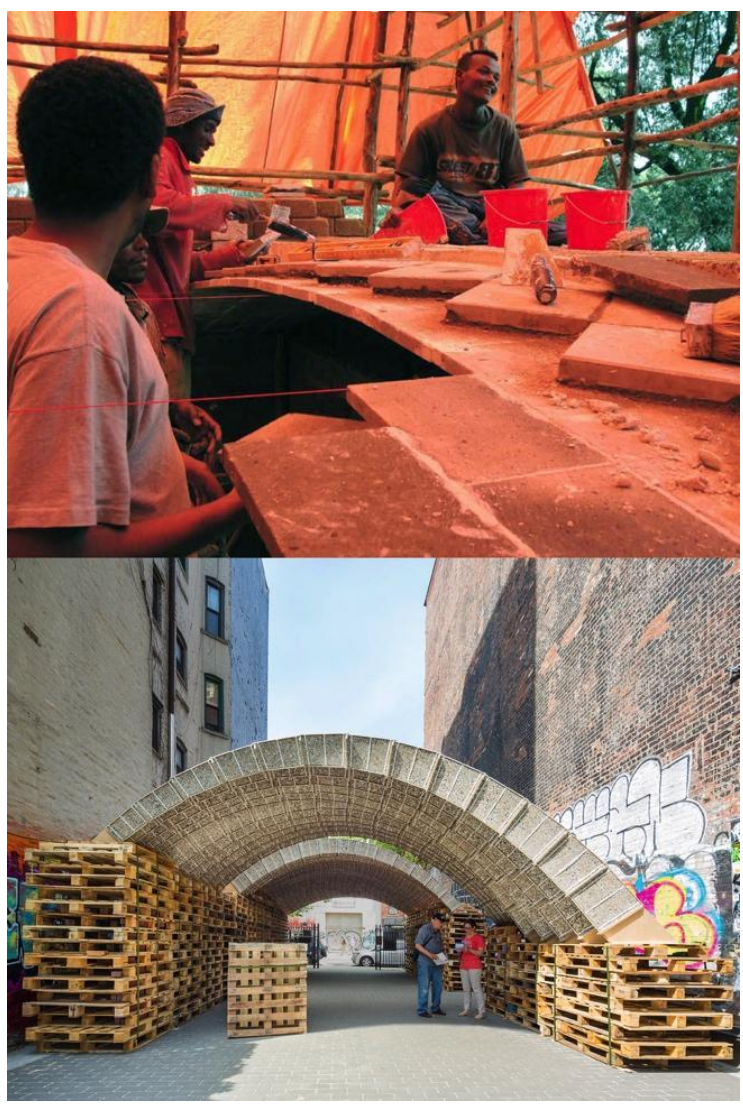

Figure 1. SUDU - the Sustainable Urban Dwelling Unit in Addis Ababa, Ethiopia (top) and WasteVault

- the ETH Pavilion at the 2015 IDEAS CITY

Festival in New York City (bottom) are both compression-only shell structures from low-strength materials

The below-described MycoTree at the 2017 Seoul Biennale of Architecture and Urbanism in Korea was built to test, validate and showcase this potential.

\section{Mycelium-bound materials}

Mycelium is the root network of fungi, a fastgrowing matrix that can act as a natural binder. It consists of individual hyphae, which grow from mycelium fungal strain spores and consume feedstock containing carbon and nitrogen [17]. Digesting plant-based waste products, such as sawdust, mycelium's dense network binds the substrate into a structurally adequate material.

The advantages of such materials as a potential alternative to traditional building materials are significant. As mycelium-bound building components are organic in matter, they can simply be composted after their original use. Furthermore, 
mycelium-bound building components can act to reverse carbon emissions through the absorption of carbon. On the other hand, the production of mycelium-bound materials, which is based on locally produced wood and agricultural waste products could address a wide range of economic, environmental and socio-cultural issues. The outcome of this research shows how mycelium can transform low-strength waste products into a high impact, affordable and sustainable material, which could be used in the construction sector.

\section{Development of mycelium-bound material for the MycoTree structure}

\subsection{Cultivation process}

Research activities at KIT and FCL focus on developing mycelium-bound building components using organic waste products as substrates. For the MycoTree, the mycelium strain Ganoderma Lucidum (G. lucidum) of Basidiomycetes, commonly known as Lingzi mushroom was chosen. This particular strain grows quickly and sturdily in a typical tropical climate. Material engineering and production of building elements for the MycoTree were conducted in collaboration with the company Mycotech in Indonesia.

The use of agricultural waste products was investigated as an alternative to substrates consisting of waste products from wood, as commonly used in the production of mycelium-bound materials. The recipe of the mycelium-bound material was developed regarding the composition of its substrate as well as the conditions of the mycelium growth aiming at maximising its compressive strength and rigidity while minimising its growth and production time. Waste from sugarcane and the tapioca starch production, specifically the roots of Manihot esculenta, more commonly known as cassava, were selected as basis for the production of the final MycoTree components.

A mycelium-bound material grown under the same conditions on a mix of woodchips and sawdust of the plant Albizia Chinensis wood species (which is widely available in Indonesia) was used as a reference for comparison.

The substrate mixtures were inoculated using pregrown spawns cultivated on corn.

Since mycelium-bound materials have only been introduced recently, no international standard currently exists that prescribes the production and testing methods for such materials. Therefore, a modified production method was developed at FCL Singapore using inputs from agricultural mushroom farms [18] and previous published works [19-21]. Briefly, the process of producing mycelium-bound material can be summarised into different phases as stated below:

- Fiberising agricultural waste products into smaller pieces;

- mixing the substrates with further supplementary nutrition;

- adjusting the water content to $60-65 \%$ in the substrate mixture;

- autoclaving the substrate in $121{ }^{\circ} \mathrm{C}$ for 30 minutes to avoid contamination by other competing microorganisms;

- inoculating the sterilized substrate with G. Lucidum spawn;

- incubating the inoculated substrate in climate conditions of $28{ }^{\circ} \mathrm{C}$ and $80 \%$ relative humidity for 8-12 days for initial colonization;

- crushing the colonised substrate and placing the mixture into moulds for further incubation in the same climate conditions for another 6-9 days;

- removing the incubation moulds and exposing the mycelium-bound material to air for chitinous skin development;

- transferring the fully grown specimens into a ventilated oven with the temperature of $80^{\circ} \mathrm{C}$ for 24-48 hours in order to reduce the moisture content to less than $10 \%$ and consequently preventing further growth.

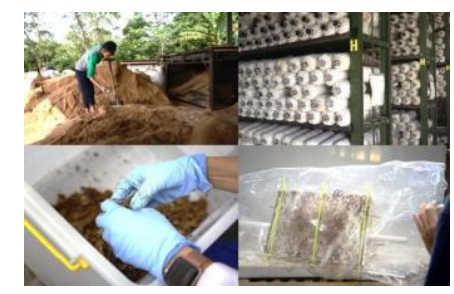

Figure 2. Production of MycoTree components: substrate collection and processing; incubation in bag logs; crushing the colonised substrate; final growth in mould

\subsection{Testing and results}

Mechanical properties of the various myceliumbound materials including compressive stress $(\sigma)$ and modulus of elasticity (E) were determined under static compressive loads using a Shimadzu $100 \mathrm{kN}$ Universal Testing Machine (UTM) and cubic specimens of $50 \times 50 \times 50 \mathrm{~mm}$. Tests were conducted according to ASTM D3574, a standard for testing flexible cellular materials [19].

Several images were taken using Scanning Electron Microscopy (SEM) to ascertain the cellular structure of the mycelium hyphae, growth patterns and the porosity of the newly developed myceliumbound materials. A JEOL 5410 SEM was used at a 
setting of $1.0 \mathrm{kV}$. The samples were coated with gold-palladium to prevent a charging effect.

6.2.1. Mechanical testing results. The compressive stress at $5 \%$ deformation of the specimens was recorded during testing as it was defined as the allowable stress limit in the structural design of MycoTree components. Table 1 displays a comparison of density, stress at $5 \%$ deformation and modulus of elasticity of two sets of mycelium-bound composite samples with two different substrate compositions: (i) sawdust and woodchips, and (ii) sugarcane and waste of cassava roots. While the first mixture represents a commonly used substrate for mycelium cultivation, the second substrate mixture was specifically developed for the components of the MycoTree.

Table 1. Physical and mechanical properties of mycelium-bound material samples with different substrate compositions

\begin{tabular}{l|c|l|l}
\hline $\begin{array}{l}\text { Substrate } \\
\text { composition }\end{array}$ & $\begin{array}{l}\text { Density } \\
\left(\mathrm{kg} / \mathrm{m}^{3}\right)\end{array}$ & $\begin{array}{l}\text { Average } \\
\text { elastic } \\
\text { modulus } \\
\text { (MPa) }\end{array}$ & $\begin{array}{l}\text { Average } \\
\text { compressive stress } \\
\text { at 5\% deformation } \\
\text { (MPa) }\end{array}$ \\
\hline $\begin{array}{l}\text { (i) woodchip and } \\
\text { sawdust }\end{array}$ & 420 & 3.97 & 0.17 \\
\hline $\begin{array}{l}\text { (ii) Sugarcane and } \\
\text { cassava roots }\end{array}$ & 440 & 22.70 & 0.61 \\
\hline
\end{tabular}

The compressive stress that developed in mycelium-bound samples grown on (i) woodchips and sawdust at $5 \%$ deformation amounts to an average of $0.17 \mathrm{MPa}$ with an average elastic modulus of $3.97 \mathrm{MPa}$. The mycelium-bound materials based on (ii) sugarcane and waste of cassava roots developed an average compressive stress of 0.61 $\mathrm{MPa}$ at $5 \%$ deformation with an average elastic modulus of $22.70 \mathrm{MPa}$, hence achieving superior material properties.

6.2.2. Scanning electron microscopy results. Samples were cut from the surface of a single mycelium-bound material for SEM imaging and were exposed to different magnifications. Figure 3a shows the SEM image of mycelium-bound materials grown on woodchip and sawdust while Figure $3 \mathrm{~b}$ shows the SEM image of mycelium-bound materials grown on sugarcane and waste of cassava roots. As shown in Figure 3, a denser network of mycelium hyphae has been observed in mycelium-bound materials grown on sugarcane and waste of cassava roots, which correlates with the higher mechanical performance reported in the previous section.
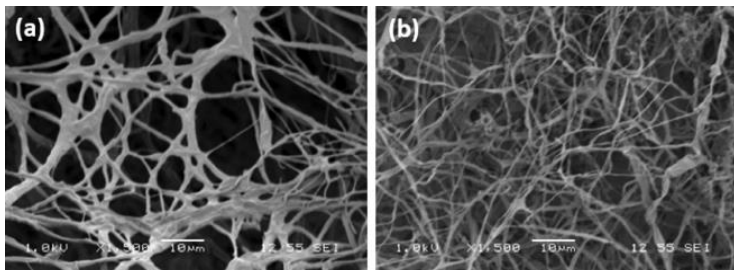

Figure 3. SEM images of the surfaces of the mycelium-bound material samples grown on substrate of: (a) woodchip and sawdust, and (b) sugarcane and waste of cassava roots $(1500 \mathrm{x}$ magnification)

\section{Structural geometry for low-strength materials}

The development of engineered materials, such as concrete or steel, is largely focused on making the materials stronger by increasing their allowable stresses. Mycelium-bound materials offer significant ecological advantages over established engineered materials on the one hand, but comparably low structural strength on the other. To build with materials that are weak in tension and bending, the use of good geometry is essential to guarantee static equilibrium of the structure through contact only, i.e., through compression. Achieving stability through geometry rather than material strength opens up the possibility of using weak materials in structural applications.

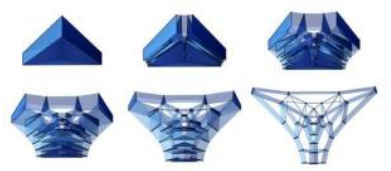

Figure 4. Exploration of structural geometry through manipulation and interactive visualisations of polyhedral reciprocal diagrams used in 3D graphic statics

The geometry of the structure was designed using 3D graphic statics, a novel method developed by the Block Research Group at ETH Zürich that extends the traditional 2D structural design technique to fully spatial systems [22]. Using polyhedral form and force diagrams, the method allows exploration and discovery of efficient and expressive spatial structures that are in compression-only, going beyond the arch or vault [23-25]. While the mycelium portion of the structure is a metaphor for a load-bearing component of a building, the grid at the top of the structure represents a floor or a ceiling of a building that applies load to the mycelium structure. 


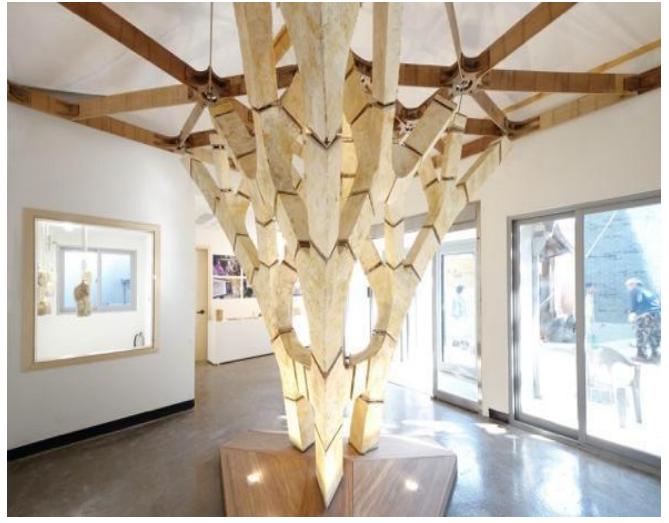

Figure 5. MycoTree is the centrepiece of the Beyond Mining - Urban Growth exhibition at the 2017 Seoul

Biennale of Architecture and Urbanism in Korea

The form finding of the structural geometry was guided by several key constraints. First, in order to minimise the geometric complexity while maximising fabricability, all nodes were limited to a valency of four, meaning there are no more than four mycelium elements coming together at any of the nodes. Second, the angle between any two linear mycelium members was constrained to be larger than 30 degrees. In addition, the centre-to-centre distances between any pair of nodes were constrained to be at least $40 \mathrm{~cm}$ such that smooth transitions could be made between any two directly adjacent nodes. Lastly, the maximum length of any linear mycelium member was limited to $60 \mathrm{~cm}$ in order to avoid potential buckling.

\section{Fabrication of MycoTree}

One of the most rewarding benefits of using 3D graphic statics to design spatial structures, is that the geometry of the structure is polyhedral by construction. Without the need for additional optimisation processes, the massing of the complex nodes of the structure can be developed through polyhedral transformations that only use planar and flat surfaces. As a result, the geometry of the moulds for the nodes could be materialized using only developable surfaces, which can be laser or $\mathrm{CNC}$ cut from readily available and easy-to-recycle sheet materials. In order to reduce the number of mechanical connections and adhesives, teeth-and-slit joints are used for the walls of the mould for efficient assembly and clean expressions of the curved edges after demoulding. Once the mould has been assembled, it can be filled with the mycelium mixture, which requires incremental compacting and additional filling as the mixture gradually densifies.

To ensure clean connections and even load transfers from one element to another in the final structure, the ends of each individual mycelium- bound components are capped with plates of bamboo composite material.
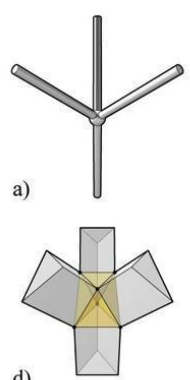

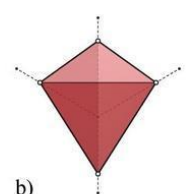

b)

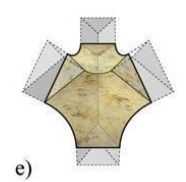

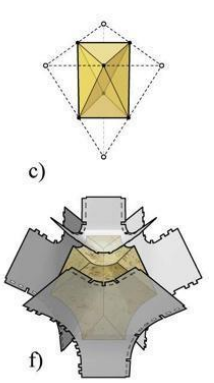

Figure 6. Development of the mould geometry: a) the orientation of linear members at a node; b) convex hull of points that are equidistant along each of the

members from the centre of the node; c) dual transformation of the convex hull; d) extrusion of the dual hull faces along the orientation of the corresponding members; e) filleting of the edges, and; f) the subsequent, developable surfaces of the node mould

These plates are also used for compacting the mycelium mixture into the moulds, and are eventually secured to the mycelium-bound components with dowels. This bamboo composite material has been developed and optimized over the past five years at FCL Singapore in order to withstand high tensile and bending forces [26].

While the mycelium components are designed to carry their self-weight plus the weight of the grid through compression, the grid on top is acting primarily in tension; it holds the branching linear mycelium elements at the top together and prevents them from falling over. For this reason, the grid is also constructed out of the bamboo composite material, utilizing $\mathrm{CNC}$-cut, $8 \mathrm{~mm}$ thick bamboo composite boards. For ease of assembly and for minimisation of steel connections, all joints of the grid are designed with slit-and-slot connections that can be easily assembled on-site and secured using simple locking elements and wooden dowels.

\section{Data and facts}

The structure supports a four by four-meter bamboo grid at a height of three meter over ground. It consists of 36 linear members of maximum $60 \mathrm{~cm}$ in length as well as 15 nodal elements of myceliumbound material. The bamboo grid weighs $134 \mathrm{~kg}$ in total, the overall weight of mycelium-bound members amounts to $182 \mathrm{~kg}$.

The triangular sections of the mycelium-bound members are sized for a limit stress value of 0.1 $\mathrm{MPa}$. The self-weight of the structure was considered with a safety factor of 1.35 during design. The structure can withstand an accidental horizontal point 
load of $0.7 \mathrm{kN}$ at a height of $1.27 \mathrm{~m}$ (arm height) from the ground at a single node. However, because the structure is designed to be primarily in compression-only, any type of horizontal loading should be avoided.

\section{Conclusion and outlook}

MycoTree is the result of a collaboration between the Professorship of Sustainable Construction at Karlsruhe Institute of Technology (KIT) and at the FCL Singapore, and the Block Research Group at the Swiss Federal Institute of Technology (ETH) Zürich. It is the centrepiece of the Beyond Mining - Urban Growth exhibition at the Seoul Biennale of Architecture and Urbanism 2017 in Seoul, Korea curated by Hyungmin Pai and Alejandro Zaera-Polo, and was be on display in Pavilion i7 at the Donuimun Museum Village from September 1st to November 5th 2017, with a 6 month extension after the exhibition closed due to increased interest and for further durability testing.

The structure represents a provocative vision of how we may move beyond the mining of our construction materials from the earth's crust to their cultivation and urban growth; how achieving stability through geometry rather than through material strength opens up the possibility of using weaker materials structurally and safely; and, ultimately, how regenerative resources in combination with informed structural design have the potential to propose an alternative to established, structural materials for a more sustainable building industry.

It will become increasingly important for building codes to adapt where necessary to allow for the potential rewards - and to address the potential challenges - of such alternative natural, recovered or cultivated materials. With world population growing at a rate of 2.47 persons per second [27], and a simultaneously decreasing supply of finite building materials stored in the earth crust, alternative concepts for the construction sector need to be implemented immediately. The material aspects of a circular economy and an innovative engineering approach need to be closely integrated to achieve this goal. As Hans Joachim Schellenhuber, Head of the Potsdam Institute for Climate Impact Research once formulated: 'The best architects in the world need to design houses made out of waste [28]'.

\section{References}

[1] Frondel, M., P. Grösche, D. Huchtemann, A.Oberheitmann, J. Peters, G. Angerer, C. Sartorius, P. Buchholz, S. Röhling and M. Wagner (2005) Trends der Angebots- und Nachfragesituation bei mineralischen Rohstoffen, Bundesministerium für Wirtschaft und Technologie (BMWi) Forschungsprojekt Nr. 09/05,
Rheinisch-Westfälisches Institut für Wirtschaftsforschung (RWI Essen), Fraunhofer-Institut für System- und Innovationsforschung (ISI), Bundesanstalt für Geowissenschaften und Rohstoffe (BGR), Germany.

[2] Hebel, D.E., M.H. Wisniewska and F. Heisel (2014) Building from Waste, Recovered Materials in Architecture and Construction. Birkhäuser, Berlin, Germany.

[3] Scrivener, K. (2015) Lecture at the chair of Marc Angelil ETH Zürich, Oerlikon, Switzerland.

[4] Milliman, J.D. and J.P.M. Syvitski (1992) 'Geomorphic/tectonic Control of Sediment Discharge to the Ocean: The Importance of Small Mountainous Rivers', The Journal of Geology, September 1, pp. 525-44.

[5] SFR TV (2014) 'ECO Spezial: Sand - ein Milliardengeschäft', SFR TV Documentary, Zurich, Switzerland.

[6] Peduzzi, P. (2014) 'Sand, Rarer than One Thinks', in Environmental Development, 2014, vol. 11, pp. 208-18.

[7] Delestrac, D. (2014) 'Sand Wars', Documentary, France.

[8] Reller A. and T.E. Graedel (2009) 'How long will it last?', Infographic, NewScientist, Great Britain; http://www.newscientist.com/data/images/archive/2605/26 051202.jpg (28 February 2018)

[9] WU Global Material Flows Database (2015) 'Global material extraction by material category, 1980-2013', materialflows.net, Vienna University of Economics and Business (WU), Vienna, Austria; http://www.materialflows.net/materialflowsnet/trends/anal yses-1980-2013/global-material-extraction-by-materialcategory-1980-2013/ (7 January 2018).

[10] Ellen MacArthur Foundation (2012) Towards the Circular Economy 1: Economic and Business Rationale for an Accelerated Transition, January 2012, Ellen MacArthur Foundation, Cowes, Isle of Wight, Great Britain.

[11] Stahel, W.R. (1982) 'Product-Life Factor', Houston Area Research Center (HARC), The Woodlands, TX, USA.

[12] Sobek, W. and H. Trumpf (2008) 'Sustainable Tall Buildings - Some Introductory Remarks', in Proceedings of CTBUH 8th World Congress, March 3-5, 2008, Dubai, UAE.

[13] McDonough, W. and M. Braungart (2002) Cradle to Cradle: Remaking the Way We Make Things, North Point Press, New York, USA.

[14] Hebel, D.E. and F. Heisel (2017) Cultivated Building Materials: Industrialized Natural Resources for Architecture and Construction, Birkhäuser, Berlin, Germany.

[15] Hebel, D.E., M. Moges and Z. Gray (2015) SUDU Research and Manual (2 Volumes), Ruby Press, Berlin, Germany. 
[16] Heisel, F. (2015) 'Waste Vault - The ETH Zürich Pavilion at the IDEAS CITY Festival in New York City', FCL Magazine Special Issue Constructing Alternatives Research Projects 2012-2015 - Assistant Professorship Dirk E. Hebel (October 2015), pp. 68-77.

[17] Carlile, M. (1995) 'The success of the hypha and mycelium', in The growing fungus. Springer, Berlin, Germany, pp. 3-19.

[18] Stamets, P. (2011) Growing gourmet and medicinal mushrooms, Ten Speed Press, Berkeley, USA.

[19] Travaglini, S., J. Noble, P.G. Ross and C.K.H. Dharan (2013) 'Mycology matrix composites', in Proceedings of the American Society for Composites - 28th Technical Conference, State College, PA, USA.

[20] Lelivelt, R., G. Lindner, P.M. Teuffel and H.M. Lamers (2015) 'The production process and compressive strength of Mycelium-based materials' in First International Conference on Bio-based Building Materials. 22-25 June 2015, Clermont-Ferrand, France. pp. 1-6.

[21] Pelletier, M., G.A. Holt, J.D. Wanjura, A.J. Lara, A. Tapia-Carillo, G. McIntyre and E. Bayer (2017) 'An evaluation study of pressure-compressed acoustic absorbers grown on agricultural by-products', Industrial crops and products, vol 95, Elsevier, pp. 342-347.

[22] Akbarzadeh, M., T. Van Mele and P. Block (2015) 'On the equilibrium of funicular polyhedral frames and convex polyhedral force diagrams', Computer-Aided Design, 63, pp. 118-28.

[23] Akbarzadeh, M., T. Van Mele and P. Block (2015) 'Three-dimensional compression form finding through subdivision', in Proceedings of the IASS Symposium 2015, Amsterdam, The Netherlands.

[24] Lee, J., T. Van Mele and P. Block (2016) 'Formfinding explorations through geometric transformations and modifications of force polyhedrons', in Proceedings of the IASS Symposium 2016, Tokyo, Japan.

[25] Lee, J., T. Van Mele and P. Block (2018) 'Disjointed Force Polyhedra', Computer-Aided Design, DOI: 10.1016/j.cad.2018.02.004.

[26] Hebel, D.E., A. Javadian, F. Heisel, K. Schlesier, D. Griebel and M. Wielopolski (2014) 'Process-Controlled Optimization of the Tensile Strength of Bamboo Fiber Composites for Structural Applications', Composites Part B: Engineering Journal, Elsevier.

[27] Umrechnung.org (2018) 'Weltbevölkerungszähler Weltbevölkerungs-Statistik', Umrechnung.org, Wünnewil, Switzerland;

https://www.umrechnung.org/weltbevoelkerung-aktuellemomentane/weltbevoelkerungs-zaehler.htm (24 February 2018).

[28] Schellenhuber, H. (2015) 'Der funktionale Slum', in Süddeutsche Zeitung, 25.4.2015, Munich, Germany.

\section{Acknowledgements}

The authors would like to thank ETH Global, the Department of Architecture at ETH Zürich, the Karlsruhe Institute of Technology and the SingaporeETH Centre for their financial support.

Furthermore, the authors would like to acknowledge all other team members who were involved in design, production and assembly of MycoTree:

- Block Research Group, ETH Zürich: Tomás Méndez Echenagucia, Andrew Liew, Noelle Paulson;

- Future Cities Laboratory, Singapore-ETH Centre: Hokie Christian, Orion Tan Sheng Yu, Kelly Cooper;

- Mycotech, PT Miko Bahtera Nusantara, Indonesia: Robbi Zidna Ilman, Erlambang Adjidarma, Ronaldiaz Hartantyo.

Finally, the authors would like to acknowledge the use of facilities for analysis, characterization, testing, and simulation at NTU, Singapore. 